\title{
Familial osteodysplasia, Anderson type
}

INSERM

\section{Source}

INSERM. (1999). Orphanet: an online rare disease and orphan drug data base. Familial osteodysplasia, Anderson type. ORPHA:2769

Familial osteodysplasia, Anderson type is a rare, genetic dysostosis disorder characterized by craniofacial bone abnormalities (i.e. midface hypoplasia, broad, flat nasal bridge, narrow, thin prognathic mandible with pointed chin, malocclusion, partial dental agenesis) associated with additional osseous anomalies, including scoliosis, calvarial thinning, pointed spinous processes, clinodactyly and abnormal phalanges. Elevated erythrocyte sedimentation rate, hyperuricemia and hypertension have also been reported. There have been no further descriptions in the literature since 1982. 